\section{A device for the easy administration of volatile anesthetics to rats undergoing stereotaxic surgery*}

\author{
LYNN T. KOZLOWSKI and STEPHEN C. WOODS \\ Columbia University, New York, New York 10027
}

Conventional procedures do not provide a practical method for the administration of volatile anesthetics such as halothane to rats while they are undergoing stereotaxic surgery. This paper describes a device which meets this need and which is easy and inexpensive to construct. A two-piece cylinder cut from the mouth of a polyethylene bottle forms a closed system around the muzzle of a rat and does not interfere with the stereotaxic apparatus or with the operation itself. The cylinder is attached to any standard device for the dispension of volatile anesthetics. The immediate advantage is that behavioral assessments can be made relatively soon after surgery.

Volatile anesthetics such as ether or halothane are often preferred to anesthetics whose duration of action is relatively uncontrollable, such as the barbiturates. But volatile anesthetics are not commonly used in conjunction with stereotaxic surgery in small animals, due to the difficulty of tracheal cannulation and hence the lack of a closed system. The present paper describes an easily constructed and inexpensive device which circumvents this problem. The device consists of a two-piece cylinder which, when placed tightly around the rat's head, forms an almost completely closed system (see Fig. 1). The arm, mounted to one of the two pieces, is attached to any standard device for the administration of volatile anesthetics (e.g., the system for halothane administration described by Luschei \& Mehaffey, 1967).

The mouth of a 1-liter polyethylene bottle serves as the cylinder, and the bottle cap, cut to provide two appropriately threaded rings, serves to hold the halves of the cylinder together when necessary. One of these threaded rings is simply a shortened

*This project was supported in part by NIH Grant 1 RO1-AM 15647-01 to S. Woods and in part by NIMH Training Grant 2T01-MH 07556-11 under which L. Kozlowsik was a trainee. surface and perpendicular to the preceding cut (see Fig. 1B). The ends of the cylinder are fitted with foam rubber to increase the tightness of fit over the stereotaxic mouthpiece at one end and the rat's muzzle at the other (see Fig. 1, C and D). The original opening of the bottle faces away from the rat. The cylinder should be long enough to cover the nose and mouth of the animal and yet not reduce the operating area on the top of the skull. (The animal's end of the cylinder can be cut at an angle providing a relatively longer ventral than dorsal wall.)

The rat is first anesthetized with the volatile anesthetic (see Luschei \& Mehaffey, 1967). The flow of the gas is then shunted to the arm of the cylinder, and the cylinder is placed around the rat's muzzle. The cylinder is held tightly by threading the shortened cap onto it. The mouthpiece of the stereotaxic instrument is removed, and the threaded ring is slipped over it. The ear bars are attached in the normal manner. The cap (Fig. 1A) and the other a threaded ring. In halving the cylinder, a zig-zag pattern is followed along its length to minimize slippage. Each cut is made at approximately a 45-deg angle from the
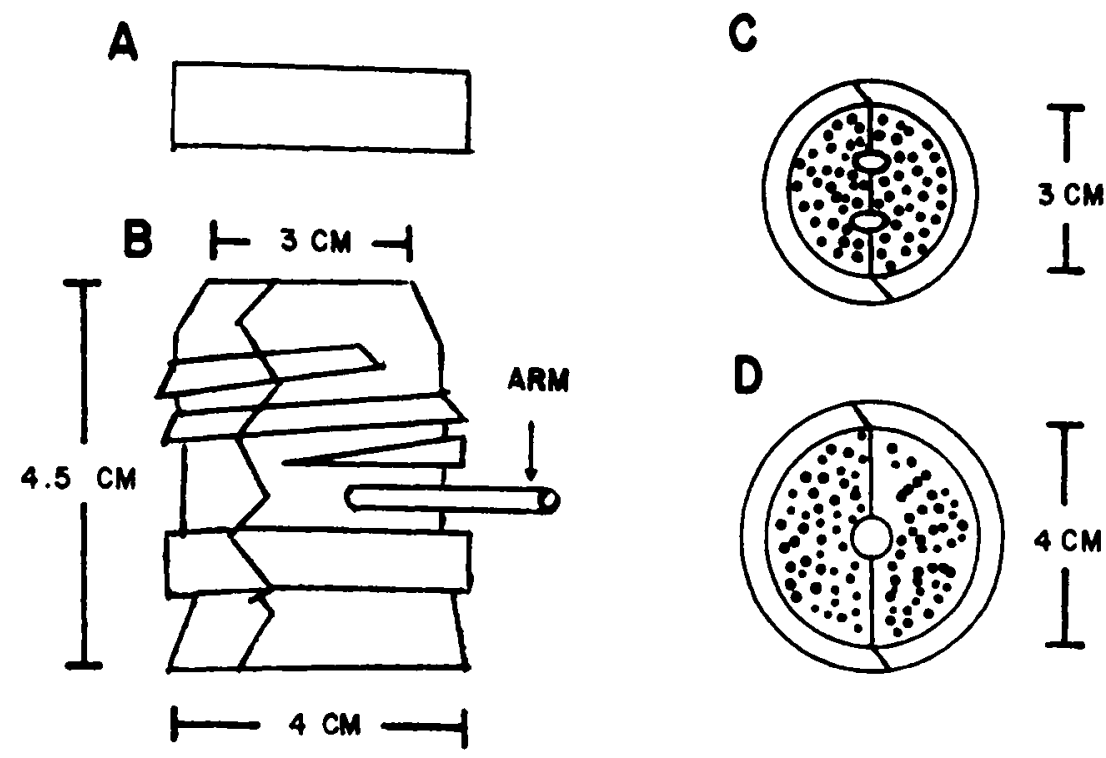

Fig. 1. Diagram of the cylinder used to administer volatile anesthetics to rats. (A) Side view of the threaded cap of the cylinder. (B) Side view of the two rejoined halves of the cylinder. (C) Top view of the cylinder. The two holes are for the mouthpiece apparatus of the stereotaxic instrument. (D) Bottom view of the cylinder. The hole is for the muzzle of the rat. The stippled areas of $C$ and D represent foam rubber. 


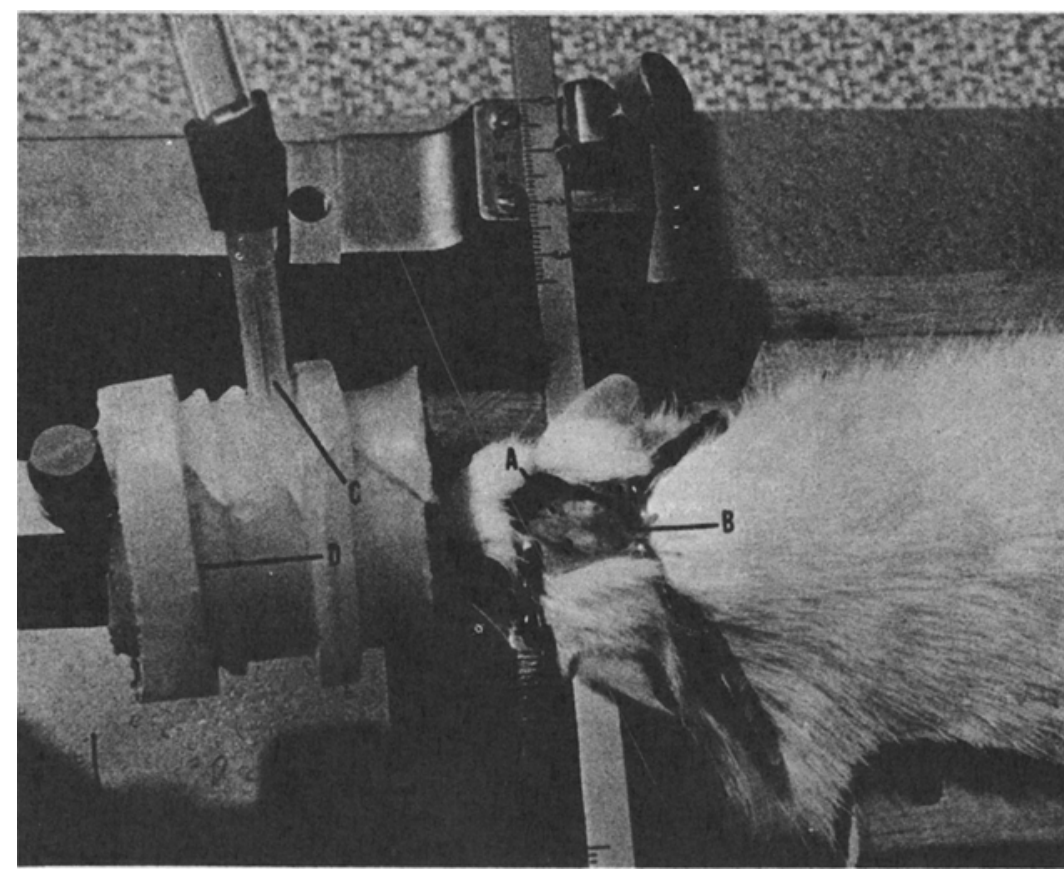

Fig. 2. Photograph of an anesthetized rat in a stereotaxic inctrument with the cylinder attached. Bregma (A) and lambda (B) are shown to demonstrate the accesability of the top of the skull. $C$ is the arm of the cylinder through which the anesthetic is administered. $D$ is the threaded polyethylene ring which holds the two halves of the cylinder together. mouthpiece apparatus is remounted on the stereotaxic instrument, the cylinder is momentarily removed from the rat, and the rat is affixed to the mouthpiece. The cylinder is then quictly repleced and joined by the threaded ring, completing the manouwer. Digure 2 show a nt in a stereotaxic indrument with the cylinder in pluce.

This devies bas been oed with halothane, and we have had sood ruces with the to for (around $150 \mathrm{~m} / \mathrm{min}$ ) and concentration (2\%-8\%) ar inated by Luchei Mehaffoy (1967), If the survary it completed and the animal's stin sutured or wound-clipped prior to removal of the cylinder, about 20 min are requined for relatively complete recovery from the anestheais. Thi time could be decreased by removnl of the cylinder at any denired stage of the operation. If immediately postoperative behavioral analyses are desired, local anesthesia of the skin on the top of the head might be desirable to minimize the discomforts of the surgery.

\section{REFERENCE}

LUSCHEI, E. S. \& MEHAFFEY, J. J. Smal animal anesthedia with halothane. Journal of Applied Physiology, 1967, 22, 695-697.

\section{ERRATUM}

TARPY, R. M. A superior method for constructing a shock grid for rats. (Vol. 4, p. 175). - Figure 2 is unfortunately upside down. We apologize. 